\title{
Síntesis y Caracterización de Espumas Flexibles de Poliuretano Obtenidas a partir de Aceite de Castor Maleinizado
}

\author{
Paula Mazo, Luis A. Rios \\ Departamento de Ingeniería Química, Universidad de Antioquia, Colombia
}

Diana Estenoz

Instituto de Desarrollo Tecnológico para la Industria Química (INTEC), Argentina

\begin{abstract}
Resumen: En este trabajo se realiza la síntesis de espumas flexibles de poliuretano empleando un diseño experimental Taguchi, utilizando aceite de castor con y sin maleinización, se estudia la cinética de la reacción de esterificación del aceite por cuantificación de los grupos ácido (ASTM D4662 - 03) y se evalúan las propiedades mecánicas de las espumas (densidad, resistencia tensil, \% elongación, resiliencia y dureza. Norma NTC 2019 Icontec) obtenidas en función de las variables de síntesis (cantidad y tipo de catalizador, relación molar aceite castor (CO) - aceite maleinizado (MACO), cantidad de agua y surfactante empleado). Se realizan análisis de FTIR y SEM para las espumas. Los resultados mostraron una cinética de primer orden con respecto al anhídrido. El aceite maleinizado (MACO) incrementa la resistencia mecánica de las espumas, debido al mayor entrecruzamiento por la incorporación de grupos ácido.
\end{abstract}

Palabras-claves: Poliuretano, espuma flexible, aceite castor, maleinización.

\section{Flexible Polyurethane Foam Synthesis and Characterization Obtained from Maleinizated Castor Oil}

Abstract: In this work, the synthesis of polyurethane flexible foam is realized with the experimental design Taguchi, using castor oil with and without maleinization. The kinetic reaction of oil esterification was monitored by estimating the number of acid groups (ASTM D4662 - 03), and the mechanical properties of the foam were studied, including density, tensile resistance, \% elongation, resilience and hardness (Norm NTC 2019 Icontec). The influence of various synthesis parameters was investigated, namely amount and type of catalyst, molar ratio of castor oil (CO) - maleinizated castor oil (MACO), amount of water and surfactant used. The foams were analyzed using FTIR and scanning electron microscopy (SEM). The results showed first order kinetics with respect to the anhydride. The maleinizated castor oil (MACO) increased the mechanical resistance of the foam due to the greater interweaving by the incorporation of acid groups.

Keywords: Polyurethane, flexible foam, castor oil, maleinization.

\section{Introducción}

El petróleo, es la materia prima para sintetizar la inmensa mayoría de los polímeros. Sin embargo esta fuente es limitada y prácticamente no renovable, y tanto los procesos involucrados en la síntesis como los materiales producidos tienen en muchos casos alto impacto ambiental. Por estas razones, actualmente se está propendiendo a la búsqueda de materas primas alternativas renovables y a la síntesis de polímeros más benignos desde el punto de vista ambiental. En este contexto, es de interés la obtención de materiales poliméricos biodegradables provenientes de aceites naturales ${ }^{[1,2]}$.
Los aceites naturales tienen la ventaja de su amplia disponibilidad y además poseen una estructura química funcional que permite sintetizar una gran variedad de productos a partir de ellos ${ }^{[3]}$.

Este es el caso del aceite de castor, el cual es monoinsaturado con grupos hidroxilo en la molécula de triglicérido, lo que ofrece la posibilidad de reacciones como epoxidación/hidroxilación ${ }^{[4-8]}$, hidroformilación ${ }^{[9]}$, glicerólisis ${ }^{[10-14]}$, ozonólisis $^{[15-17]}$, entre otras. Estas reacciones se pueden explotar para la obtención de materiales poliméricos tales como los poliésteres, los poliuretanos y las poliamidas ${ }^{[18]}$.

Los poliuretanos se obtienen comúnmente empleando dioles de alto peso molecular derivados del petróleo. Con

Autor para correspondência: Paula Mazo, Grupo Procesos Fisicoquímicos Aplicados, Departamento de Ingeniería Química, Universidad de Antioquia, 
la crisis energética y los problemas asociados al desabastecimiento de esta materia prima se han comenzado a emplear polioles de aceites vegetales como fuentes alternativas, tales como el castor y soja modificada, con la ventaja adicional de bajar el impacto ambiental de los procesos de síntesis. Las espumas flexibles a base de aceite de castor presentan deficiencias debido a la alta rigidez y dureza que presentan.

Las espumas flexibles de poliuretano (PU) poseen celdas abiertas, son permeables al aire, son reversibles a la deformación y pueden ser producidas con diversas propiedades incluyendo firmeza y resiliencia.

La primera producción de este tipo de espumas, basadas en la reacción entre un isocianato aromático y un poliol poliéster, fue llevada a cabo en 1954. Sin embargo, esas espumas no soportaron las condiciones severas de humedad y temperatura a las cuales fueron sometidas. Para solucionar estos inconvenientes fueron desarrollados los polioles polieter, cuyas espumas suministraron mejor durabilidad y mejor confort. Desde entonces ha aumentado el desarrollo de formulaciones para diversas aplicaciones que requieren mayor resistencia ${ }^{[19]}$. Han sido reportados muchos trabajos de investigación en la síntesis de poliuretanos basados en aceite de ricino o castor Lu et al. ${ }^{[20]}$, Rodrigues ${ }^{[21]}$, Costa y Duarte ${ }^{[22]}$, Keyur et al. ${ }^{[23] \text {, }}$ Siddaramaiah y Mallua ${ }^{[24]}$, Ajithkumar et al. ${ }^{[25]}$, Mazo et al. ${ }^{[26]}$, pero muy pocos estudios se han realizado sobre la modificación del aceite mediante maleinización. Bertz et al. ${ }^{[27]}$ y Hong et al. ${ }^{[28]}$, modificaron el aceite para aplicaciones cosméticas y espumas flexibles entrecruzadas con estireno.

El presente trabajo se fundamenta en un estudio experimental con análisis estadístico utilizando el método Taguchi en un sistema de poliuretano empleando MACO. Los objetivos del trabajo fueron: estudiar la cinética de la reacción de esterificación del aceite de castor con anhídrido maléico y evaluar las propiedades mecánicas de las espumas formadas variando la composición de los reactivos empleados en la síntesis.

\section{Experimental}

\section{Materiales y métodos}

Para las reacciones se emplearon los siguientes reactivos: aceite de castor grado USB, con un valor de $\mathrm{OHv}=159,51 \mathrm{mgKOH} / \mathrm{g}$ muestra, determinado mediante titulación con agente ftalante, anhidrido maleíco grado comercial (Merk), 2,4 Toluen diisocianato (TDI) grado comercial (Merk), silicona con un porcentaje de siloxano de $25-35 \%$
(General Electric) y un peso molecular de 6000, agua corriente sin ningún tratamiento, dimetanol amina grado comercial (Tego), octoato de estaño grado comercial (Tego), tolueno grado analítico (Merk).

\section{Diseño experimental}

El diseño experimental utilizado fue un diseño $\mathrm{L}_{9}$ de cuatro factores con tres niveles (Tablas 1 y 2) elaborado por el método Taguchi y se utilizó para evaluar la influencia de las variables en las propiedades mecánicas de las espumas obtenidas. El método Taguchi es un diseño robusto que tiene su origen en las ideas del ingeniero japonés Genichi Taguchi que combina métodos de ingeniería y estadística para obtener mejoras en los costos y la calidad de los productos ${ }^{[29]}$.

La relación molar de grupos isocianato a grupos hidroxilo (NCO:OH) permanecerá constante en un valor de 1,3.

La selección de los niveles utilizados para cada una de las variables, se estableció de acuerdo a formulaciones base reportadas en la literatura, donde la cantidad de poliol requerida se mantiene constante y la cantidad de isocianato se calcula mediante la relación de los equivalentes estequiométricos requeridos para reaccionar con todos los compuestos donadores de protones que se encuentran en la formulación, entre ellos están los polioles, extensores de cadena, aminas, agua, etc.

Las variables respuesta para estos experimentos fueron: resiliencia, dureza, \% elongación, resistencia a la tensión y densidad aparente, determinados mediante la norma NTC 2019 ${ }^{[30]}$.

Los espectros IR-ATR se realizaron en un espectrofotómetro marca Perkin Elmer y la morfología de la superficie de las espumas fue observada en un microscopio de escaneo de electrones (scanning electron microscope. SEM) Hitachi S-520. Las espumas fueron cortadas en capas de $2 \mathrm{~mm}$ de espesor y recubiertas con una capa de oro.

\section{Síntesis y caracterización del aceite de castor modificado}

El anhídrido maléico y el aceite de castor fueron adicionados en una relación molar 1:1 en un reactor de $250 \mathrm{~mL}$. Se mantuvo mediante agitación variando el tiempo de 0 a 8 horas, bajo atmosfera de nitrógeno. Las temperaturas de reacción fueron 80,87 y $100{ }^{\circ} \mathrm{C}$. Se tomaron muestras a lo largo de la reacción para determinar el valor ácido por titulación (ASTM D4662 - 03) y el número de hidroxilos (ASTM D4274 - 05).

Tabla 1. Factores y niveles a analizar en la producción de una espuma flexible de poliuretano.

\begin{tabular}{|c|c|c|c|c|}
\hline & & \multicolumn{3}{|c|}{ Nivel } \\
\hline & & 1 & 2 & 3 \\
\hline \multirow[t]{4}{*}{ Factor } & A. Relación molar CO:MACO & $75: 25$ & $50: 50$ & $25: 75$ \\
\hline & B. Cantidad de agua & 3,0 & 3,5 & 4,0 \\
\hline & C. Cantidad de catalizador (Octoato de estaño) & 0,1 & 0,5 & 1,0 \\
\hline & D. Cantidad de amina (Dimetanolamina) & 0,1 & 0,5 & 1,0 \\
\hline
\end{tabular}


Tabla 2. Diseño de experimentos para la producción de una espuma de PU.

\begin{tabular}{cccccc}
\hline \multicolumn{5}{c}{ Indice NCO/OH: 1,3 } & \multirow{2}{*}{ Respuesta } \\
\cline { 1 - 4 } Arreglo interno L ${ }_{\mathbf{9}}$ & \\
\cline { 1 - 4 } Exp/nivel & A & B & C & D & Yij \\
\cline { 1 - 4 } 1 & 1 & 1 & 1 & 1 & Y1 \\
2 & 1 & 2 & 2 & 2 & Y2 \\
3 & 1 & 3 & 3 & 3 & Y3 \\
4 & 2 & 1 & 2 & 3 & Y4 \\
5 & 2 & 2 & 3 & 1 & Y5 \\
6 & 2 & 3 & 1 & 2 & Y6 \\
7 & 3 & 1 & 3 & 2 & Y7 \\
8 & 3 & 2 & 1 & 3 & Y8 \\
9 & 3 & 3 & 2 & 1 & Y9
\end{tabular}

*Factores A: relación molar CO:MACO; B: cantidad de agua; C: cantidad de catalizador (octoato de estaño); D: cantidad de amina (dimetanolamina); y total de experimentos $=9$ con tres réplicas cada uno.

\section{Preparación de las espumas de poliuretano flexible}

Se mezclan $\mathrm{CO}$ y MACO a temperatura ambiente, en una cantidad total de $100 \mathrm{pph}$ (partes por cien); luego se incorpora el catalizador octoato de estaño, silicona, isocianato, agua y amina mediante agitación constante. Las cantidades de los reactivos se describen en las Tablas 1 y 2 , la cantidad de isocianato se calcula de acuerdo a la cantidad estequiométrica de grupos $\mathrm{OH}$ del aceite.

\section{Resultados y Discusión}

La Figura 1 muestra los espectros IR del aceite maleinizado (MACO) a diferentes conversiones, las muestras se codifican de acuerdo con el valor de hidroxilos obtenido empleando una temperatura de $100^{\circ} \mathrm{C}$. Puede observarse que el pico a $3450 \mathrm{~cm}^{-1}$ correspondiente al estiramiento de grupos $\mathrm{OH}$, disminuye al aumentar el tiempo de reacción, el pico a $1644 \mathrm{~cm}^{-1}$ que puede asignarse al doble enlace $\mathrm{C}=\mathrm{C}$, aumenta. No se observan picos a 1779 y $1849 \mathrm{~cm}^{-1}$, correspon- dientes a anhídridos cíclicos, por lo cual el anhídrido está reaccionando con los hidroxilos del aceite.

A partir de los datos experimentales de la Tabla 3, se calculo el porcentaje de conversión de acuerdo a la Ecuación 1, se realizó un estudio cinético de la disminución del grado de acidez a fin de determinar la velocidad de la reacción de esterificación, usando un modelo de regresión lineal se determinaron los parámetros de la ecuación de Arrhenius de la reacción de esterificación y los parámetros termodinámicos asociados utilizando la teoría del estado de transición.

$$
\eta=\left(\frac{A v_{o}-A v_{f}}{A v_{o}}\right) * 2
$$

donde $\eta$ es el porcentaje de conversión, $\mathrm{Av}_{\mathrm{o}}$ acidez inicial, $\mathrm{Av}_{\mathrm{f}}$ acidez final.

La reacción siguió una cinética de primer orden y los parámetros cinéticos obtenidos se muestran en la Tabla 4.

Los parámetros termodinámicos para el estado de transición calculados a $100{ }^{\circ} \mathrm{C}(373.15 \mathrm{~K})$ se muestran en la Tabla 5. Los cálculos se realizaron basados en la teoría del estado de transición y mediante la ley de Arrhenius, donde:

$$
\begin{aligned}
& E_{a}=\Delta H^{\neq}(T)+R T \\
& A=e k_{B} T / h \exp \left[\Delta S^{\neq}(T) / R\right] \\
& \Delta G^{\neq}=\Delta H^{\neq}-T \Delta S^{\neq}
\end{aligned}
$$

$\mathrm{E}_{\mathrm{a}}$ es la energía de activación, $\mathrm{k}_{\mathrm{B}}$, h, y R son las constantes de Boltzman, Planck y la de los gases universal. $\Delta \mathrm{G}^{\neq}(\mathrm{T})$ es la energía libre estándar de activación, $\Delta \mathrm{H}^{\ddagger}(\mathrm{T})$ es la entalpía estándar de activación y $\Delta \mathrm{S}^{\neq}(\mathrm{T})$ es la entropía estándar de activación a la temperatura absoluta $\mathrm{T}$.

En la Tabla 5 se muestra que: el signo del cambio en la entalpía para el estado de transición $\left(\Delta \mathrm{H}^{\ddagger}\right)$ representa un estado endotérmico y un estado de transición tardío, donde ocurren importantes cambios en la polaridad reactivos/TS. El cambio de entropía para el estado de transición $\left(\Delta \mathrm{S}^{\mp}\right)$ tiene signo positivo

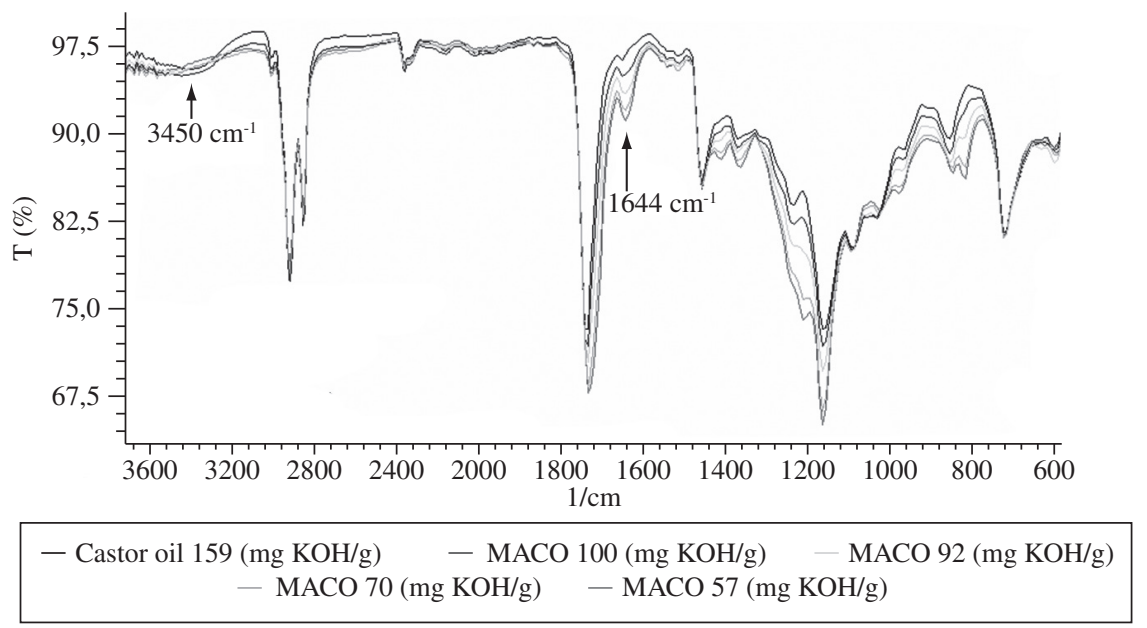

Figura 1. Espectros IR de las muestras de aceite maleinizado. 
Tabla 3. Valor de acidez (AV) para las muestras de aceite maleinizado.

\begin{tabular}{|c|c|c|}
\hline Tiempo horas & Temperatura $\left({ }^{\circ} \mathbf{C}\right)$ & AV \\
\hline 0 & 80 & 108,76 \\
\hline 0,5 & 80 & 100,12 \\
\hline 1 & 80 & 92,23 \\
\hline 2 & 80 & 87,15 \\
\hline 3 & 80 & 82,21 \\
\hline 4 & 80 & 76,34 \\
\hline 5 & 80 & 66,43 \\
\hline 6 & 80 & 61,32 \\
\hline 7 & 80 & 60,46 \\
\hline 8 & 80 & 59,78 \\
\hline 0,5 & 87 & 90,65 \\
\hline 1 & 87 & 78,12 \\
\hline 2 & 87 & 75,23 \\
\hline 3 & 87 & 73,32 \\
\hline 4 & 87 & 70,45 \\
\hline 5 & 87 & 69,50 \\
\hline 6 & 87 & 61,02 \\
\hline 7 & 87 & 58,06 \\
\hline 8 & 87 & 56,91 \\
\hline 0,5 & 100 & 80,23 \\
\hline 1 & 100 & 64,45 \\
\hline 2 & 100 & 63,32 \\
\hline 3 & 100 & 62,45 \\
\hline 4 & 100 & 61,07 \\
\hline 5 & 100 & 60,09 \\
\hline 6 & 100 & 58,87 \\
\hline 7 & 100 & 57,47 \\
\hline 8 & 100 & 55,01 \\
\hline
\end{tabular}

Tabla 4. Parámetros cinéticos para la reacción de esterificación del aceite de castor con anhídrido maléico sin catalizador.

\begin{tabular}{ccccc}
\hline $\mathbf{T}(\mathbf{K})$ & $\mathbf{k}\left(\mathbf{m i n}^{-1)}\right.$ & $\mathbf{r}^{2}$ & $\mathbf{E a}\left(\mathbf{J} . \mathbf{m o l}{ }^{-1}\right)$ & $\mathbf{A}\left(\mathbf{m i n}^{-1}\right)$ \\
\hline 353,15 & 0,322 & 0,969 & 11714,426 & 17,69 \\
360,15 & 0,362 & 0,907 & - & - \\
373,15 & 0,401 & 0,857 & - & - \\
\hline
\end{tabular}

Tabla 5. Parámetros termodinámicos para la esterificación.

\begin{tabular}{|c|c|c|}
\hline$\Delta \mathbf{H}^{\ddagger}\left(\mathrm{J}^{\prime} \mathrm{mol}^{-1}\right)$ & $\Delta \mathbf{S}^{ \pm}\left(\mathrm{J}^{\prime} \mathrm{Kmol}^{-1}\right)$ & $\Delta \mathbf{G}^{\neq}\left(\mathrm{J}^{\prime} \mathrm{mol}^{-1}\right)$ \\
\hline 8612,057 & 188,852 & $-63746,39$ \\
\hline
\end{tabular}

lo que indica una disminución del orden en el estado de transición debido al aumento en las colisiones moleculares, el cambio en la energía libre de Gibs indica un proceso espontáneo.

La reactividad y la energía liberada por la reacción con el diisocianato disminuye al disminuir el valor de hidroxilos del aceite, esto es debido a que disminuyen los grupos reactivos del poliol. Además se incrementa el efecto estérico debido al entrecruzamiento con el anhídrido maléico. Esto es muy conveniente a nivel industrial ya que disminuye el riesgo de reacciones con exotermia incontrolable y autocombustión que se generan en la obtención de espumas con aceite de ricino sin modificar.

A partir de los valores obtenidos para las propiedades mecánicas de las espumas mostrados en las Tablas 6 y 7, se realizó un análisis mediante gráficas de efectos (los cuales no se muestran en este trabajo por razones de espacio). Se puede determinar que los factores seleccionados afectan significativamente al valor medio para todas las propiedades mecánicas evaluadas. En general, el nivel 2 es el recomendado para disminuir los valores de densidad y dureza, aumentar la resistencia tensil, elongación y resiliencia, sin embargo debe realizarse un diseño más refinado para establecer cual seria las cantidades y condiciones óptimas.

Al aumentar la cantidad de aceite maleinizado o modificado (MACO) en las formulaciones comparado con una espuma de polieter convencional (Tabla 7), aumenta la densidad debido a que el nivel de catalizador de estaño es muy alto y produce encogimiento y reducción de volumen en las espumas, afectando su valor, sin embargo los grupos acido pueden reaccionar con el isocianato y generar $\mathrm{CO}_{2}$, lo que favorecería la espumación a un nivel de catalizador adecuado. La resistencia tensil y el porcentaje de elongación aumentan debido a que se produce un mayor entrecruzamiento por la incorporación de grupos ácido los cuales son mas reactivos que los hidroxilos secundarios, pero a su vez se aumenta la fase amorfa o segmentos suaves en el poliuretano aumentando flexibilidad del polímero, la resiliencia del material que es la capacidad de absorber energía en el periodo elástico disminuirá, aumentando el rebote y la dureza.

La Figura 2 muestra un espectro IR de las espumas obtenidas variando la relación de $\mathrm{CO}$ y MACO, donde puede observarse que: no existen diferencias significativas entre los espectros, solo en el caso de la espuma obtenida con MACO

Tabla 6. Propiedades mecánicas de las espumas obtenidas, valores promedio.

\begin{tabular}{cccccc}
\hline Experimento & Densidad $\left(\mathbf{K g} / \mathbf{m}^{3}\right)$ & Resistencia tensil $(\mathbf{K P a})$ & Elongación (\%) & Resiliencia (\%) & Dureza (N) \\
\hline 1 & 34,55 & 224,0 & 91,5 & 5,82 & 36,73 \\
2 & 32,73 & 164,6 & 82,6 & 9,71 & 46,13 \\
3 & 82,24 & 387,6 & 85,6 & 5,82 & 41,43 \\
4 & 116,26 & 406,2 & 135,9 & 7,67 & 41,43 \\
5 & 48,73 & 388,4 & 239,2 & 5,82 & 46,13 \\
6 & 30,00 & 194,9 & 96,8 & 9,71 & 39,08 \\
7 & 42,55 & 151,6 & 85,6 & 5,82 & 38,14 \\
8 & 31,79 & 192,0 & 101,7 & 9,94 & 39,08 \\
9 & 34,61 & 166,4 & 90,0 & 11,65 & 36,73 \\
\hline
\end{tabular}


$100 \%$, muestra que la banda a $3287 \mathrm{~cm}^{-1}$ correspondiente con el estiramiento NHCOO, del grupo uretano que a su vez puede solaparse con la banda NHCO de los grupos urea y amida, se aumenta debido a la formación de amida por la reacción de los grupos ácido de MACO con el isocianato, la banda a 1729,1640 y $1170 \mathrm{~cm}^{-1}$, correspondientes con los estiramientos del carbonilo para los grupos uretano y urea, también aumentan.
La Figura 3 muestra las micrografías de las espumas con diferente composición de $\mathrm{CO}$ y MACO, los diámetros de celda fueron determinados de acuerdo al método ASTM 3576-98, la incorporación de MACO en la formulación aumenta el tamaño de las celdas comparada con la espuma obtenida de ricino $100 \%$, si se quiere aumentar el tamaño y disminuir la densidad, se debe adicionar un agente espumante adicional.

Tabla 7. Propiedades mecánicas de las espumas obtenidas, valores promedio.

\begin{tabular}{lccccc}
\hline \multicolumn{1}{c}{ Experimento } & Densidad $\left(\mathbf{K g} / \mathbf{m}^{3}\right)$ & Resistencia tensil $(\mathbf{K P a})$ & Elongación $(\boldsymbol{\%})$ & Resiliencia $(\boldsymbol{\%})$ & Dureza $(\mathbf{N})$ \\
\hline 100 Aceite & 32,35 & 128,30 & 59,90 & 11,65 & 39,08 \\
100 Aceite modificado & 41,35 & 185,00 & 102,30 & 11,65 & 32,03 \\
Polieter convencional & $27,1-31$ & $70-140$ & $70-145$ & $20-40$ & $16-25$ \\
\hline
\end{tabular}

Experimentos realizados a 3,5 pph agua, 0,5 pph octoato de estaño, 0,5 pph dimetanol amina.

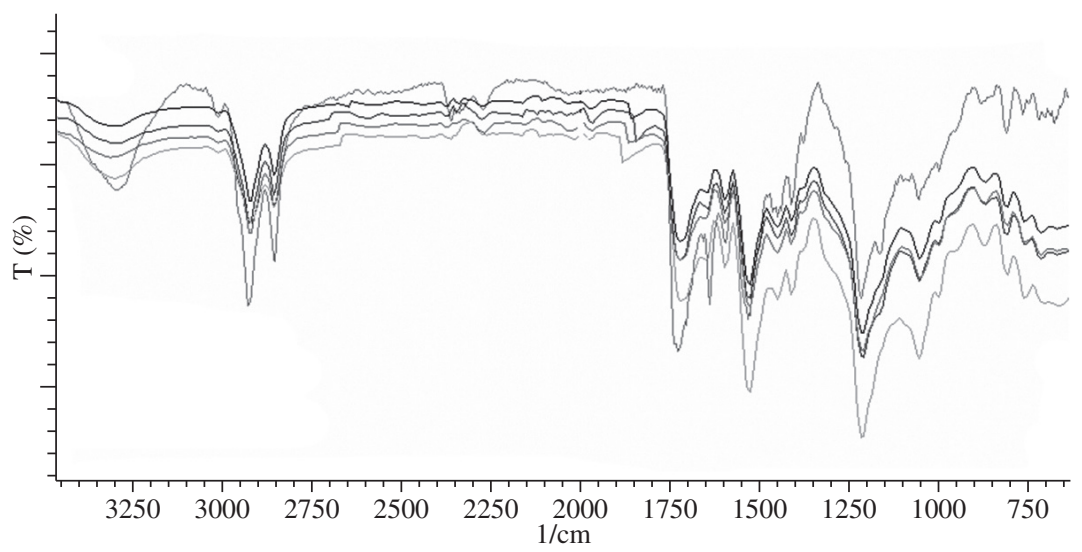

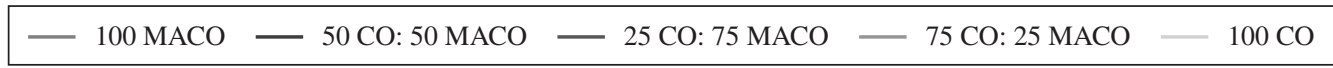

Figura 2. Espectros IR de las muestras de espumas flexibles de PU.

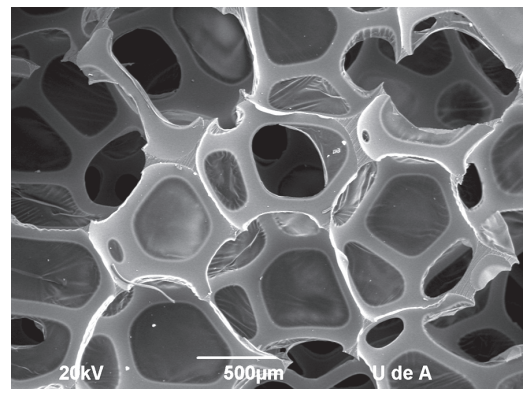

(a)

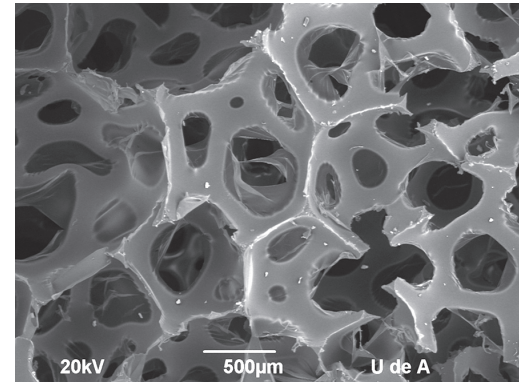

(b)

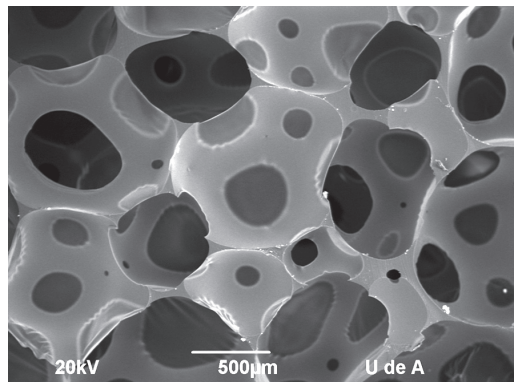

(c)

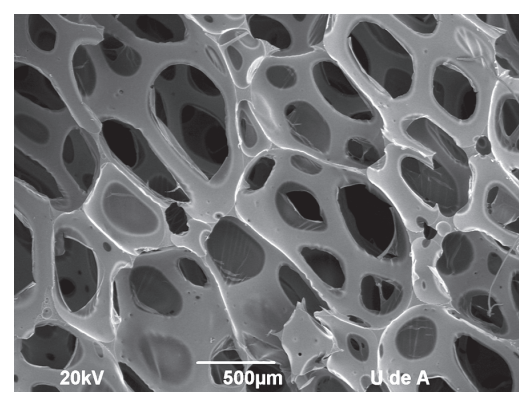

(d)

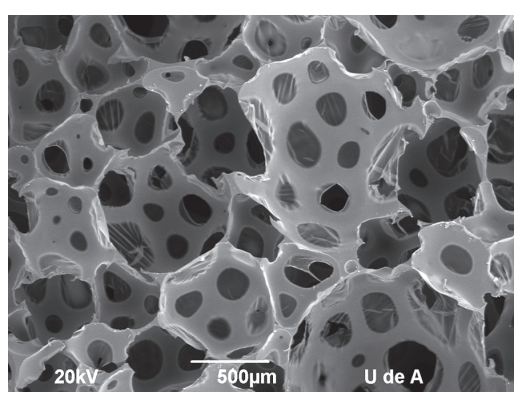

(e)

Figura 3. Morfologia de las espumas de PU obtenidas con diferentes composiciones de CO: MACO. a) Espuma comercial tipo polieter, diametro de celda $=649,2 \mu \mathrm{m}$; b) espuma $100 \mathrm{CO}$, diámetro de celda $=324,6 \mu \mathrm{m} ;$ c) espuma $75 \mathrm{CO}: 25 \mathrm{MACO}$, diámetro de celda $=649,2 \mu \mathrm{m} ; \mathrm{d})$ espuma 50 CO:50 MACO, diámetro de celda $=324,6 \mu \mathrm{m}$; e) espuma $100 \mathrm{MACO} 7-2$, diámetro de celda $=649,2 \mu \mathrm{m}$. 


\section{Conclusiones}

- Aunque la metodología Taguchi ha generado gran controversia por ser un método robusto que involucra una gran cantidad de variables y cuyas interacciones pueden no ser consideradas, en este trabajo se presenta como una buena alternativa para obtener una formulación de espuma que cumpla con los requisitos mínimos para las aplicaciones que requieren alta durabilidad (colchones, silletería, etc);

- Las espumas que incluyen dentro de su formulación el poliol de castor modificado, aumentan la resistencia tensil, el porcentaje de elongación y la dureza, pero disminuye el \% de resiliencia, comparada con el poliol convencional; y

- Las reacciones de obtención de espuma con ricino modificado, presentan menor exotermia y las reacciones son más lentas que las que se obtienen con el aceite de ricino sin modificar, lo cual permite tener un mejor control de la reacción.

\section{Referencias}

1. Woods, G. W. - "The ICI polyurethanes book", JohnWiley, Netherlands (1987).

2. Carme, M.; Babb D. \& Ryan, A. - Polymer., 49, p.3279-3287 (2008).

3. Mihail, I.; Zoran, S. \& Petrovic, X. - J Polym Environ., 15, (4), p.237-243 (2007).

4. Campanella, A.; Fontanini, C. \& Baltanás, M. - Chemical Engineering Journal., 144, (3), p.466-475 (2008).

5. Dinda, S.; Patwardhan, A.; Goud,V. \& Pradhan, N. - Bioresource Technology., 99, (9), p.3737-3744 (2008).

6. Goud,V.; Patwardhan, A.; Dinda, S. \& Pradhan, N. Chemical Engineering Science., 62, (15), p.4065-4076 (2007).

7. Goud,V.; Patwardhan, A. \& Pradhan, N. - Bioresource Technology., 97, (12), p.1365-1371 (2006).

8. Guidotti, M.; Ravasio, N.; Psaro, R.; Gianotti, E.; Coluccia, S. \& Marchese, L. - Journal of Molecular Catalysis A: Chemical., 250, (1-2), p.218-225 (2006).

9. Kandanarachchi, P.; Guo, A. \& Petrovic, Z. - Journal of Molecular Catalysis A: Chemical., 184, (1-2), p.65-71 (2002).

10. Tanaka, R.; Hirose, S. \& Hatakeyama, H. - Bioresource Technology, 99, (9), p.3810-3816 (2008).

11. Cheirsilp, B.; Kaewthong, W. \& H-Kittikun, A. - Biochemical Engineering Journal., 35, (1), p.71-80 (2007).
12. Pawongrat, R.; Xu, X. \& H-Kittikun, A. - Food Chemistry., 104, (1), p.251-258 (2007).

13. Corma, A.; Bee Abd Hamid, B.; Iborra, S. \& Velty, A. Journal of Catalysis., 234, (2), p.340-347 (2005).

14. Corma, A.; Iborra, S.\& Primo, J. - Journal of Catalysis., 173, (2), p.315-321 (1998).

15. Narine, S.; Kong, X.; Bouzidi, L.\& Sporns, P. - J. Am. Oil Chem. Soc., 84, p.65-72 (2007).

16. Narine, S. S.; Kong, X.; Bouzidi, L. \& Sporns, P. - J. Am. Oil Chem. Soc., 84, p.55-63 (2007).

17. Narine, S. S.; Yue, J. \& Kong, X. - J. Am. Oil Chem. Soc., 84, p.173-179 (2007).

18. Ogunniyi, D. - "Castor oil: A vital industrial raw material". Bioresource Technology., 97, p.1086 (2006).

19. Vilar, W. - "Química y Tecnología de Poliuretanos". www.poliuretanos.com.br. Tercera edición. Río de Janeiro. (2002).

20. Lu, Y.; Tighzert, L.; Dole, P. \& Erre, D. - Polymer., 46, p.9863 (2005).

21. Rodrigues, J. M.; Pereira, M. R.; Souzac, A. G.; Carvalhoc, M. L.; Dantas, A. A.; Dantas, T. N. C. \& Fonseca, J. L. C.- Thermochimica Acta., 427, p.31 (2005).

22. Costa, R. \& Duarte, M. - Progress in Organic Coatings., 51, p.6 (2004).

23. Keyur, P.; Somania, S.; Natvar, K. \& Animesh, K. - International Journal of Adhesion \& Adhesives., 23, p.269 (2003).

24. Siddaramaiah, P. \& Mallua, A. - Polymer Degradation and Stability., 63, p.305 (1999).

25. Ajithkumar, S.; Kansara, S. \& Patel, N.K. - Eur. Polym. J., 34, (9), p.1273 (1998).

26. Mazo, P.C.; Franco, A.; Cardeño, F.; Ríos, L. \& Echeverri, D. "Síntesis y caracterización de espumas de poliuretano utilizando aceites vegetales", XIV Congreso Colombiano de Química, Armenia-Quíndio, (2006).

27. Bertz, S.; Miksza, F. \& Zucker, E. "High purity adduct of castor oil and maleic anhydride". United States Patent., 6, (225), p.485, (2001).

28. Hong, J.; Wang, M.; Ming, Q.; Jing H.; Hui W. \& Tibor, C. -Biomacromolecules., 9, p.615 (2008).

29. Gutierrez, H. \& Salazar, R. "Análisis y diseño de experimentos", Mc Graw Hill, México (2004).

30. "Plásticos. Espumas flexibles de poliuretano". Norma Técnica Colombiana. NTC 2019, ICONTEC (2005).

Enviado: 11/12/08

Reenviado: 25/02/09

Aceito: 09/03/09 